\title{
Handwriting difficulties in Developmental Coordination Disorder (DCD)
}

SpringerLink Header: Motor Disorders (P Wilson, Section Editor)

${ }^{1}$ Barnett, A.L. and ${ }^{2}$ Prunty, M.

${ }^{1}$ Corresponding author: Anna L. Barnett, BA, BSc, PhD, CPsychol. Department of

Psychology, Health \& Professional Development, Oxford Brookes University, Headington Campus, Oxford, OX3 0BP, UK. abarnett@brookes.ac.uk

${ }^{2}$ Mellissa Prunty, BSc, MSc, PhD, Division of Occupational Therapy, Brunel University London 
Keywords: Legibility; speed; pauses; automaticity; letter formation; assessment

\begin{abstract}
Purpose of review. Handwriting is a particular difficulty in DCD. Children who struggle to produce legible and sufficiently fast handwriting may under-achieve at school. Using van Galen's model, this review examines recent research to describe the nature of handwriting difficulties in DCD. The range of assessment tools is highlighted and recommendations for intervention provided.

Recent findings. Embedded in the broader skill of writing, handwriting involves more than motor skill. Children with DCD tend to produce less writing than their peers. Their slow rate of production is characterised by frequent pauses. Errors in letter formation negatively impact on legibility and the quality of written composition is also poor. Different types of assessment help to capture the range of difficulties.

Summary. Comprehensive assessment helps gain a full understanding of the nature of handwriting difficulties in DCD. International recommendations and guidelines provide a valuable framework for assessment and principles for intervention.
\end{abstract}




\section{Introduction to DCD}

Developmental Coordination Disorder (DCD) is included in the DSM-5, where it is described as a difficulty with tasks that involve motor control and coordination (1). There is now a large body of research contributing to our understanding of this condition (2) which affects around $2-6 \%$ of school-aged children (3). Although international recommendations are available to guide assessment and intervention in DCD (4), there remains a lack of awareness of the condition by health and educational professionals (5). Individuals with DCD struggle to acquire and perform everyday movement tasks required at home, at school, at play/leisure and in the workplace. Handwriting in particular is an area of difficulty and is often the reason for referral to an Allied Health Professional (6). DCD commonly occurs alongside other neurodevelopmental disorders affecting attention, language and reading (e.g. ADHD, DLD, dyslexia), which themselves may impact on writing performance. This review draws on research evidence and current guidelines to: outline the nature of handwriting difficulties in DCD, indicate the range of tools available for a comprehensive assessment and provide suggestions for planning intervention.

\section{Handwriting as an Important Component of Writing}

Handwriting is not just a motor skill and is a crucial component of the overall task of writing. Alongside spelling, handwriting forms the lower level transcription skills, which govern early writing development (7). When transcription skills are laboured, valuable working memory resources are consumed resulting in reduced capacity to engage with higher-level writing processes such as planning and revision $(8,9)$. As such, the quality of the written composition can be impacted by poor handwriting skill as less attention is available to focus on key components including organisation of ideas, sentence structure, grammar, punctuation, vocabulary and spelling (10). Indeed the link between handwriting speed (typically the number of words produced per minute) and the quality of written composition has been demonstrated in numerous studies on children with $(11,12)$ and without $(13)$ handwriting difficulties including those with DCD (14). Handwriting itself is a complex skill. Unlike most other motor skills, it is intricately linked to language and there are a number of cognitive and linguistic processes that occur before, during and after the pen touches the page. While some models of handwriting focus specifically on the biomechanical motor components (e.g. $(15,16)$, Van Galen's (1991) (17) psychomotor model of handwriting is the only one to also include the broader cognitive and linguistic processes (see Fig. 1). Although first published almost three decades ago, the hierarchically arranged cognitive, linguistic and motor 'processing modules' provide the most comprehensive model available. Each processing level in the model is associated with the writer working on particular output 'units'. These units decrease in size, from a broad idea of what to write to a specific stroke of the pen on the page. Although some more recent research has suggested possible additions to the linguistic processes of van Galen's model (see (18)), even in its original form it serves as a useful framework for understanding handwriting and is therefore used to structure this review.

\section{Handwriting Difficulties in Children with DCD}


According to van Galen (1991), the first level in the handwriting process is the activation of the intention to write. This largely involves the writer's motivation to engage with the task. Rosenblum, Margieh and Engel-Yeger (19) examined this in a group of children with DCD through two specific questions from the Handwriting Proficiency Screening Questionnaire (HPSQ; (20)). Teachers reported a higher incidence of reluctance to write in the DCD group and less engagement with homework compared to peers. However, studies on goal setting in children with DCD would suggest that many children want to improve their handwriting as part of their goals for therapy $(21,22)$. It is therefore important to note that while there may be a reluctance to write in this group, this may not reflect the motivation to improve.

Assuming the intention to write has been activated, the writer then generates ideas at the level of semantic retrieval. This refers to accessing ideas and concepts from the verbal lexicon. These are translated into language necessary for sentence structure at the level of syntactical construction. Both of these levels have been shown to be affected in the handwriting of children with Developmental Language Disorder (DLD) (11) which co-occurs with DCD in approximately $33 \%$ of cases (23). Compared to typically developing and language matched peers, children with DLD produced fewer words per minute driven by their difficulties with language (11). While no published study has looked at handwriting skill in children with DCD and DLD as a dual diagnosis, one has to assume that an overlap in these two conditions would add a layer of complexity in the expression of their difficulties with handwriting. However, further research is needed to investigate this in more detail.

Following syntactical construction, the level of spelling is activated, which involves mapping the sound of the letter to its visual representation. This is a complex process closely linked to handwriting speed, as the linguistic make up of a word has been found to dictate the pace at which it is written. For example, research by Kandel et al (24) has demonstrated that words which contain two or more syllables or are complicated to spell, take longer to process and produce compared to shorter, more frequently used words. It is therefore unsurprising that children with co-occurring dyslexia and $\mathrm{DCD}(\mathrm{DCD}+)$ demonstrate greater difficulties with handwriting compared to children with DCD or dyslexia only (25). Using digitising writing tablets, which provide temporal data on the process of handwriting, Sumner at al. found that children with $\mathrm{DCD}+$ pause for a greater percentage of time in writing tasks compared to other groups (DCD only, dyslexia only and age-matched controls). Indeed spelling ability alone impacts on handwriting performance independent of motor skill (25) and it is therefore no surprise that children with overlapping conditions experience greater difficulties.

The level of spelling is the initial stage of motor planning in handwriting, as the sound of the letter (the phoneme) closely maps to the set of instructions for producing it (the grapheme). This phoneme-to-grapheme conversion occurs at the level of the allograph. This includes the patterns of movement necessary for letter formation and depending on whether the handwriting is to be joined, un-joined or formed using capitals, the movement patterns will vary. It is worth noting that during a 10 -minute free-writing task, $65 \%$ of children with DCD $(n=33)$ from 11 different schools in the UK produced completely un-joined handwriting compared to $25 \%$ of their peers (26). While the reasons for this are unclear it is interesting that even with the emphasis on joining in the UK school curriculum (27) the majority of 
children with DCD chose a less complex style of handwriting. One factor may be the cognitive cost associated with handwriting production in this group and the need to simplify letter formation in order to cope with writing demands. Indeed research at the level of the allograph would support this notion as the process of letter formation is significantly impacted in children with DCD. Using similar methods to Sumner et al, Prunty et al. (28, 29) examined handwriting performance in English speaking children with DCD in the absence of reading and spelling difficulties. Temporal analyses of their handwriting revealed a tendency to pause for a greater percentage of writing tasks compared with typically developing peers (28). This pausing behaviour offers some insight into the slowness in production so commonly reported in this group. The pauses also appear in the handwriting of children with DCD in other languages and writing systems including Hebrew (30) and Arabic (19).

The location of pauses in the writing of children with DCD tends to be within illegible words (29). Figure 2 illustrates pausing behaviour in the handwriting of a 10 year old boy with DCD and a typically developing aged matched peer. Here the dysfluency in writing seems to be related to a lack of automaticity in letter formation as the child pauses/hesitates frequently within words. This within word pausing is characteristic of a novice/less skilled writer and is not present in the handwriting of the typically developing child who is able to produce words without hesitation . Prunty and Barnett $(31,32)$ examined issues with letter formation in children with DCD in detail by replaying the children's handwriting in real time and coding their letters for errors. Both studies revealed that children with DCD produced a higher percentage of errors in letter formation compared with typically developing peers. The most common errors included incorrect start position and strokes formed in the wrong direction (32). These letter formation errors indicate difficulties at the allograph level and ultimately impact on speed of production. In order to produce handwriting at speed it is important to move across the page efficiently, but when letters are formed with directional errors it disrupts the fluency of movement.

The majority (65\%) but not all children with DCD demonstrate incorrect letter formation (32). Some demonstrate difficulties at the next level of size control, where the writer needs to control the size of letters under task demands such as writing quickly or generating freewriting text when all of the processes of writing are at play. Difficulties with size control can constrain aspects of legibility such as spacing between words and letters, letter alignment and letter formation (i.e. too big, too small). These have all been shown to be significantly impacted in children with DCD compared to age matched controls, regardless of language or writing system $(30,33)$. However some of these aspects of legibility may also be impacted by the final process in the handwriting cycle which is the level of muscular adjustment, where the message is sent from the brain to the effectors resulting in the real time movement of the pen. While research has shown that children with DCD can move the pen just as quickly as their typically developing peers (26), the movement is more variable and less consistent (34). Bo et al (34) reported inconsistencies in the temporal aspects of movement during single letter formation in children with DCD. However, given that the linguistic make up of words has an impact on the temporal production of handwriting (24), future research would need to 
include word level analyses in order to fully understand the role of muscular adjustment in this group.

\section{Individual Differences and performance profiles}

The most recent research on handwriting difficulties in children with DCD has indicated a disruption in text production evident in pausing behaviour. As outlined above, these pauses are largely attributed to difficulties at the allograph level. However, while group data suggest that children with DCD perform below typically developing peers on measures of handwriting speed, letter formation and legibility, it is important to note the heterogeneity of this group. Two studies examining individual participant data outlined the profiles of performance in children with DCD across a range of handwriting tasks $(26,31)$. The profiles included measures of legibility, handwriting speed (and other temporal aspects such as the percentage of pausing) as well as quality of letter formation. The analyses revealed that not all children with DCD have difficulties with handwriting although the majority do (up to 95\% (26)) and while legibility is below typically developing peers in most children with DCD, handwriting speed is not always affected. In the data available, $57 \%$ of children with DCD had difficulties in all our measures (26). The severity of difficulties were varied, with some performing below 1 standard deviation (SD) of the typically developing group mean while others were 3-6 SDs below $(26,31)$. Given the variability within and between measures, clinicians must use more than one assessment to avoid overlooking a child with difficulties.

\section{Handwriting and how to assess it}

In this section, we consider the range of assessments that may be used to identify and describe handwriting difficulties in DCD. These include direct observation, questionnaires, rating scales and standardized performance tests, some of which are specifically recommended in the latest international consensus on DCD (4). We describe a selection of tools, some relating to the cognitive and linguistic processes of van Galen's model and some to the motor planning and execution processes.

\section{Cognitive and linguistic processes}

Considering the initial stages of cognitive and linguistic stages in van Galen's model, the first step in understanding handwriting difficulties is to assess attitudes towards and motivation for handwriting. Classroom teachers are well placed to assess this through informal observation of children with DCD. Observations can be recorded more formally in questionnaires. For example, in the HPSQ (20), the teacher rates (on a 5-point scale) the frequency with which particular characteristics are seen. This includes item 5 'Does the child often feel he/she does not want to write?' Inter-rater reliability on this item is reported as good (ICC 0.71). Children's own perspectives can also be gained using the child version, the HPSQ-C (35), which includes similar items about the motivation to write. A significant moderate correlation $(r=.51)$ has been reported between the child and teacher version, with children tending to rate themselves more poorly overall than their teachers. 
The next step in van Galen's model is to know what to write and to be able to retrieve semantic and syntactic information prior to producing writing on the page. The ability to do this will depend on the particular requirements of the task. In copying tasks, the content is provided. However, for many classroom-writing tasks the child is required to generate their own ideas and the appropriate syntactic structures for the text. One way to evaluate the child's capability at this stage of the writing process is to compare text produced orally with that produced in writing, both in terms of the quantity produced but also the quality (36). More formal tests of oral expression are included in broader achievement tests, such as the Wechsler Individual Achievement Test $-3^{\text {rd }}$ UK Edition (WIAT-III UK; (37) A handwriting assessment in DCD should take into account whether the child has broader language and writing difficulties (e.g. Dyslexia, DLD), which are likely to impact on the ability to generate the content for writing.

\section{Motor planning and execution}

Subsequent processes in van Galen's model involve motor planning and motor execution, as seen in Fig. 1. First the child needs to retrieve an appropriate sequence of letters to produce each word. Spelling difficulties are likely to impact on the speed of production but also word choice and consequently the quality of composition $(12,14)$. Information on the extent and nature of any spelling difficulty is therefore an important part of a general writing assessment. Difficulty with spelling may be obvious from observing a child's written text. However, since those with spelling difficulties may avoid using words that they struggle to spell, more formal evaluation can be helpful. In the UK, the SpLD Assessment Standards Committee (SASC, www.sasc.org.uk) recommend spelling tests included in the WIAT-III UK, Wide Range Achievement Test $5^{\text {th }}$ Edition (WRAT 5; (39)) and British Ability Scales $3^{\text {rd }}$ Edition (BAS3; (40)). Spelling difficulties can also have an impact on readability or legibility of the text. Understanding the nature and extent of the spelling difficulty will help intervention planning.

The next stage in the writing process is to select the individual letters or allographs. Allographs then need to be produced using the correct patterns, with an appropriate control of size and using appropriate muscular adjustment for each letter stroke, to produce letterforms that are accurate and consistent. Letter formation is the main predictor of overall handwriting legibility $(41,42)$. This includes several components such as the shape, size, closure and alignment (on the base line) of letter bodies and formation of the ascenders, descenders and letter joins. These aspects can be judged in real time by observation of the handwriting process or later through examination of the completed script. Direct observation confers some advantages as it reveals aspects of the handwriting process that might not be obvious from the final product alone. For example, the start and end position of letter formation and the consistency of movement patterns used to form letters. As mentioned above, digitising graphics tablets have been used in research for the detailed examination of handwriting in real time. These are not yet widely used for general assessment purposes but may play a role in the future. In a less formal way, some smart phone and tablet applications can record the sequence of letter strokes produced by a child and provide feedback in relation to their accuracy. 
Formal measurement of handwriting legibility is included in some standardised handwriting tools. For example, the Systematic Detection of Writing problems (SOS-2-EN; (43)), which was developed in The Netherlands and translated into English. This test involves copying increasingly complex sentences for five minutes. The script is scored according to the number of sentences judged to have errors in the following aspects: letter formation, writing fluency, connecting strokes (joins) and spacing. Transparent templates are also used to score other aspects, such as letter height. Scores from these criteria are summed to provide a total score; moderate inter-rater reliability (ICC 0.77) and test-retest reliability (ICC 0.69 ) are reported. The SOS-2 provides detailed information but scoring is time consuming and takes some effort to learn. It also needs modifications across languages and scripts. A tool that can assess legibility across different writing tasks, languages and scripts is the Handwriting Legibility Scale (HLS; (33)). Designed for quick, practical use, the rater examines a piece of handwritten work and considers overall legibility, the amount of effort required to read the script, layout/organisation of the writing, letter formation and alterations. Each aspect is rated on a 5-point scale and summed to give a total score. The HLS is reported to have acceptable inter-rater reliability (ICC 0.92, Kappa 0.67) and discriminates between the handwriting of children with and without DCD. The HPSQ and HPSQ-C, as more general questionnaires on handwriting, also include items related to legibility (e.g. asking whether the writing can be read by the child, or read by another person). These items have high inter-rater reliability (ICC 0.90 and above) for the teacher version.

Another important element of handwriting is the speed of production. If a child struggles to keep up with the writing demands of the classroom or produces too little to adequately display their knowledge in a written examination, then they may be at risk of underachievement. It is therefore important that allographs and words can be produced accurately (legibly) and at an appropriate speed. Aspects of handwriting speed are included in the HPSQ and HPSQ-C (e.g. with the item 'Does the child not have enough time to copy tasks from the blackboard?'). However, more objective and detailed assessment tools are also available. The Detailed Assessment of the Speed of Handwriting (DASH; (44)) provides a profile of performance across four main tasks: copying in 'best' handwriting, copying quickly, alphabet writing and free writing for 10 minutes. Scores are summed to give a total score, which has good test-retest reliability (with correlations above 0.80 ). Good discrimination between children with and without special educational needs and between different age groups is also reported in the test manual. Speed is also measured in the SOS-2-EN for a copying task only. This has moderate test-retest reliability (ICC 0.66) and good discrimination between typically developing children and children in special education, and between different age groups is reported for an early version of the test (45). As different writing tasks have different demands, speed must be measured separately for each.

A comprehensive assessment will include a combination of different methods to gain a full picture of a child's individual handwriting capabilities. In addition, it should be noted that the child's performance will be influenced by the particular task demands and the environment in which they are writing. For example, the language being written (and whether this is the 
child's first or an additional language), the style of writing adopted by a school, instructions for neatness or speed, a requirement to copy or to self-generate text all have different demands. A child's performance may also be influenced by the size and shape of furniture or the positioning, type and quality of paper or the type of pen/ pencil used. For example, research has found that when there is little friction between the pen and the writing surface (i.e. using a stylus on a tablet screen) the amount of control at the level of muscular adjustment is reduced. This presents as faster and less accurate movements compared to writing on paper (46). These ergonomic aspects are an important part of any assessment and should be noted when observing a child write. Since the goal of handwriting is to communicate information, a final consideration in assessment is the content of handwritten work (quality of composition). This can be reviewed informally for aspects such as sentence structure, grammar and vocabulary use. More formal assessment tools include the written expression component of the Wechsler Individual Achievement Test - Third UK Edition (WIAT-III UK; (37)).

Using a recognised model of handwriting production helps to keep assessment focused on the relevant aspects of the task. This task-oriented approach is highlighted in the most recent recommendations for assessment and intervention approaches in the field of DCD (4). Caution should be taken with other approaches to assessment that are not aligned with evidence-based frameworks or current recommendations. For example, it is sometimes suggested that shape copying should be included in a handwriting assessment, using a tool such as the Beery-Buktenica Developmental Test of Visual-Motor Integration (VMI-6; (47)). Although this might be relevant for young children, there is no evidence to support its use for the understanding of handwriting difficulties in later school aged children with DCD (48). In addition, examination of biomechanical factors in children with DCD, such as handgrip strength, pressure exerted on the page (48) and pen grasp (49) has shown no clear relationship with their handwriting difficulties. It seems the cognitive, linguistic and motor planning processes described in this review are more pertinent factors.

In choosing any assessment tool it is important to consider its practical application and psychometric properties, to ensure it is suitable and fits the purpose of assessment. Aspects of reliability and validity should be examined, as well as whether appropriate norms are available. One reason for assessing handwriting is to gather information to help devise a suitable intervention to support an individual to develop their skills.

\section{Intervention}

Current international recommendations (4) and best practice principles (50) should be consulted and followed when planning interventions for children with DCD. It is recommended that a profile of the child's strengths and weaknesses and their environmental context should be taken into account, as suggested in the above section on assessment. In addition, individualised goal setting is recommended, capturing the views of the child as well as their family and relevant others (e.g. teachers). Tools such as the PEGS-2 (51) or 'Here's how I write' assessment (HHIW; (52) can help to determine child goals. 
In planning interventions for handwriting, the focus should be on activity and participationoriented approaches (4). This means working on the task of handwriting itself, rather than addressing component skills (e.g. grip strength and visual perception). Although there has been little research to specifically evaluate handwriting interventions in DCD, some relevant information can be drawn from recent meta-analyses of handwriting instruction (53) and from the application of well-known motor learning principles (54).

One approach to the delivery of handwriting interventions is the Partnering for Change (P4C) school-based occupational therapy model. The P4C model, developed by Missiuna and colleagues $(55,56)$, involves a partnership between therapists, teachers and parents in creating a learning environment that facilitates participation. Originally developed for children with DCD, it has three levels of intervention, the first being 'universal' which involves strategies that are useful for all children but essential for some. An example of universal intervention for handwriting would include a classroom teacher demonstrating correct letter formation to the whole class. Since young children with DCD (5 years of age) form letters containing errors, explicit teaching in the early stages of learning may help mitigate this issue, which seems to be a key contributor to handwriting difficulties in this group.

The second level of the $\mathrm{P} 4 \mathrm{C}$ model includes intervention at the targeted level for children who need additional input (differentiated instruction) over and above usual teaching practices. Here, interventions that include self-evaluation/meta-cognition can be effective for handwriting (53). An example of targeted intervention for handwriting would include the Cognitive Orientation to Daily Occupational Performance (CO-OP; (57)) which can be delivered in a small group setting.

The CO-OP encourages the child to pay attention to the features of their handwriting and use self-evaluation (guided discovery) to problem solve and improve their handwriting performance. The CO-OP can also be used in a one-to-one situation where a child may need more specific input at the third level of P4C. This is known as the accommodation level where students need to be supported in a different way. In the case of handwriting, there may be multiple difficulties at play, all of which could independently impact on handwriting production. Given that DCD co-occurs with other disorders, it is likely that a child may present with not only difficulties with motor control but also the other levels in van Galen's model such as generating ideas for writing (semantic retrieval), construction of language (syntactical construction) and/or spelling. It is therefore useful to consider van Galen's model in structuring intervention. As limited working memory resources govern the whole writing process, it is not possible to focus on all areas at once. However, one way of addressing multiple difficulties in a strategic way could be to adapt and control the demands of the task. For example in the initial stages of letter formation the child could practice individual letters (level of the allograph) using blocked practice followed by combinations of letters which frequently occur together (bi-grams, tri-grams) using random practice. As handwriting improves, the child can move to practicing simple words that are easy to spell followed by writing simple sentences from dictation. By ensuring the child continues to be challenged at an appropriate level or 'challenge point' (58), this enables the child to develop 
correct letter formation in a task that controls the demands of language. As the child progresses, the generation of text (semantic retrieval) and construction of language (syntactical construction) can be introduced using cloze or simple stories. It is important to note that given the complex nature of writing, improvements in the child's handwriting will take time (at least 20 sessions) (59) and while practice sessions can be short (10 minutes) they need to be frequent (3-5 times per week). Over time, the child will continue to refine their skills and eventually should enter the autonomous stage (54). In order to support this process it is imperative that key personnel are aware of the plan for intervention including the teacher and the child's parents/primary carers in order to support the child in developing consistent strategies for writing which are positively reinforced and supported over time.

\section{The future}

While research surrounding handwriting difficulties in children with DCD has progressed in recent years, there are clear gaps in the literature that need to be addressed moving forward. First, further application of digital capture methods, both in research and in the classroom, should allow more detailed examination of the process of handwriting to further unravel the nature of inaccurate and dysfluent performance. Second, the development of robust tools to consider all aspects of handwriting are needed to provide more holistic assessments and thus better understanding of the nature of the difficulties experienced. This must include practical tools for use in the classroom with different groups of children, including those learning in a second/additional language. Third, more needs to be done to understand how children with DCD learn how to write, how much practice they require and what type of practice works best. Outcomes need to be considered not just for handwriting but also for writing composition, the quality of the text produced and ultimately academic performance. Lastly, future work in these areas should be applied with children writing in different languages and using different scripts.

\section{Conclusions}

Our review shows how examining handwriting in the broader context of writing, employing theoretical frameworks and capitalizing on digitising technology have all contributed to a better understanding of handwriting difficulties in DCD. Low output levels and poor legibility seem to be related to dysfluencies and a lack of automaticity at the allograph level. However, there is individual variation in the particular areas of difficulty experienced by children. Evidence-based guidelines and agreed international recommendations have driven developments in assessment and intervention. Assessment should consider all components of the writing process to help understand an individual profile and plan appropriate support. Universal approaches to intervention have the greatest efficiency and reach, while more targeted support will be required for some children. Task-oriented approaches will be most effective, drawing on motor learning principles and working with the child to set goals and evaluate their own performance. Future work should consider handwriting in different contexts and determine the optimal conditions for learning to enable children with DCD to achieve their potential. 


\section{Compliance with Ethics Guidelines}

\section{Conflict of Interest}

Anna Barnett reports personal fees from Pearson Assessment outside the submitted work; and being co-author of Movement ABC-2 Test and Checklist, DASH (Detailed Assessment of Speed of Handwriting) and DASH17+, all assessment instruments used in the field of DCD. Mellissa Prunty declares no conflicts of interest relevant to this manuscript.

\section{Human and Animal Rights and Informed Consent}

This article does not contain any studies with human or animal subjects performed by any of the authors. 


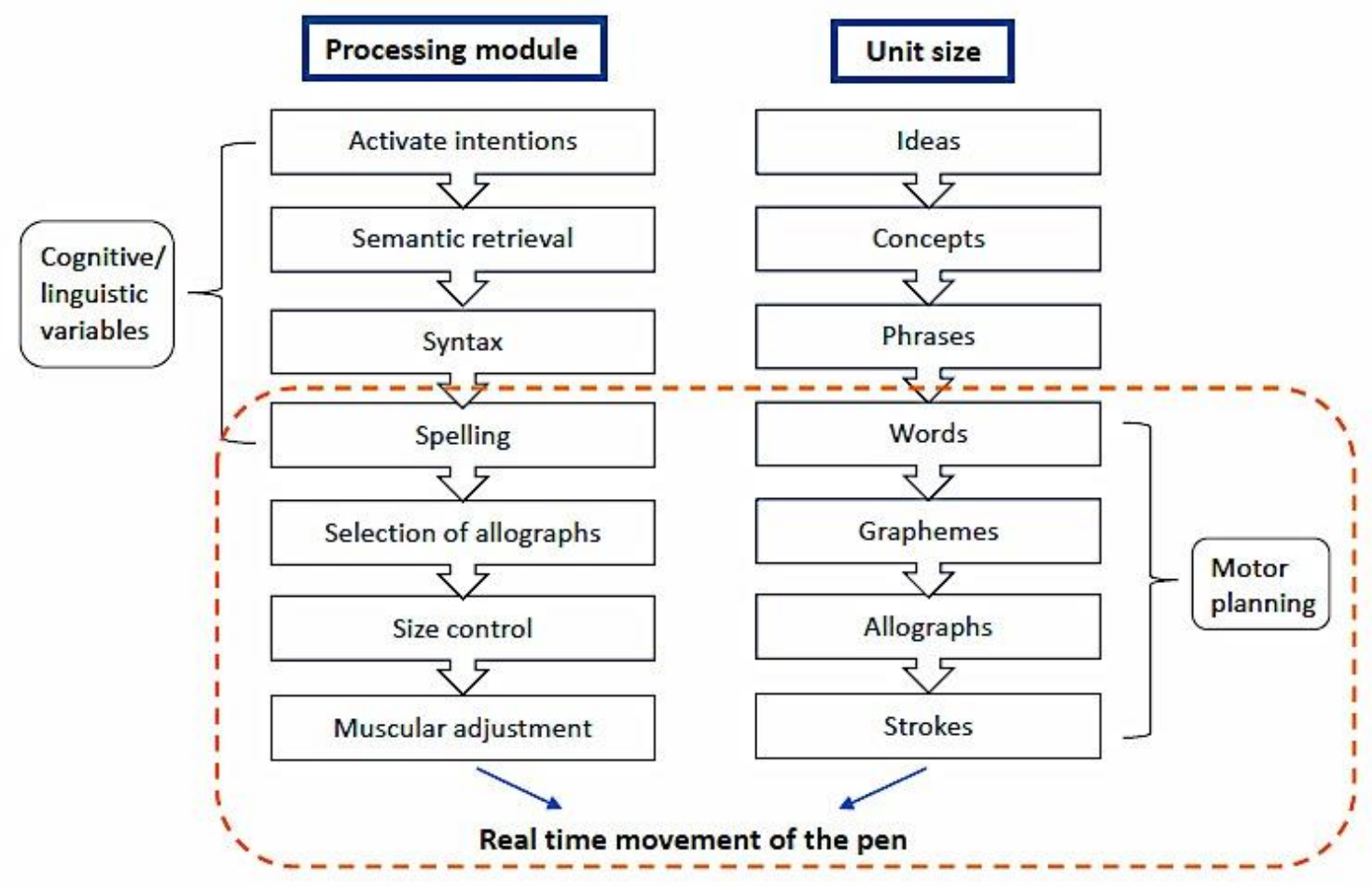

Fig. 1 Adaptation of van Galen's (1991) psychomotor model of handwriting. Processing modules activated hierarchically, moving from cognitive and linguistic processing to motor programming. Reprinted from Human Movement Science, Vol. 10/Issue 2-3, Gerard P. van Galen, Handwriting: Issues for a psychomotor theory, Pages 165-191, Copyright (1991), with permission from Elsevier. 

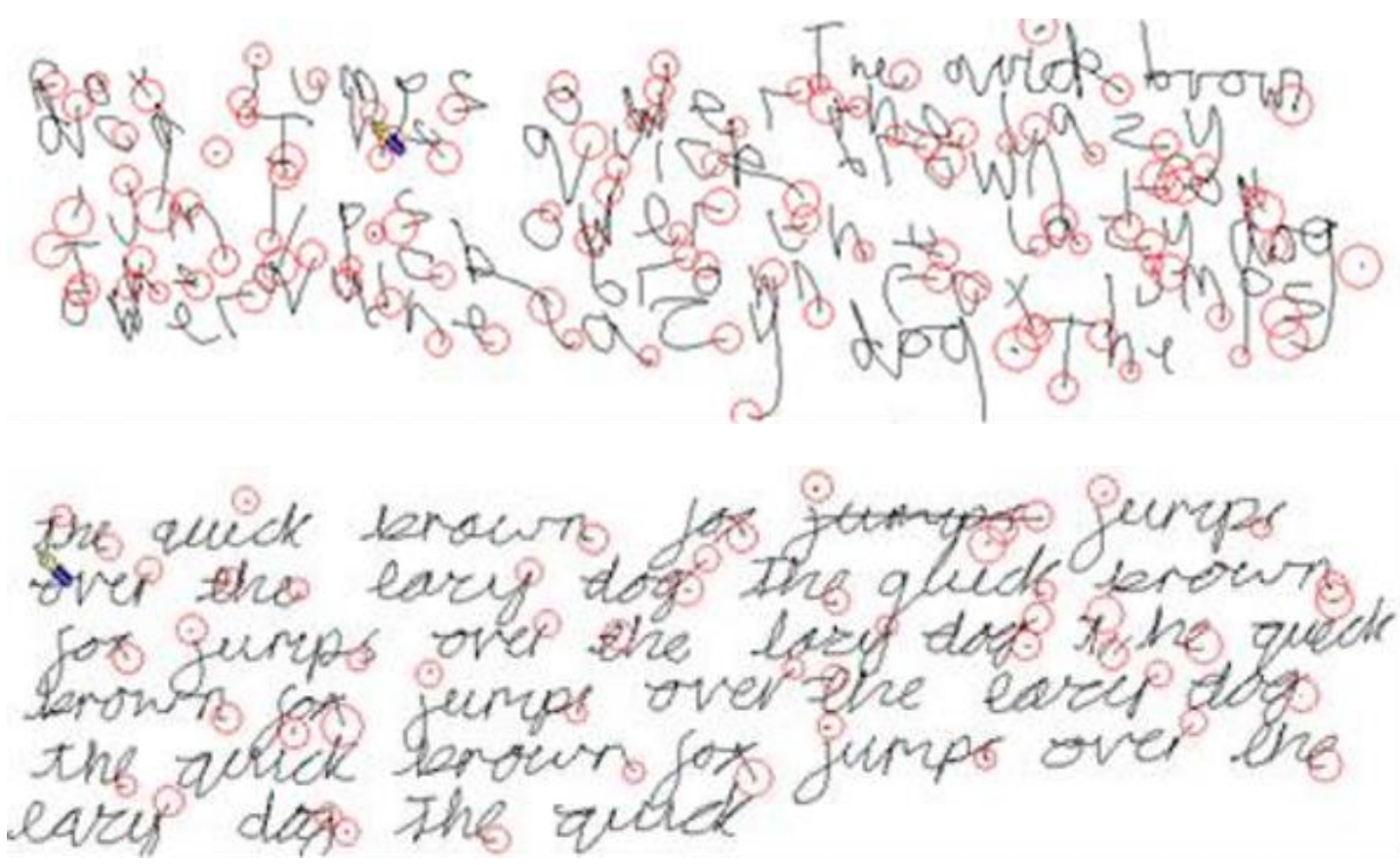

Fig. 2 Pausing behaviour in the handwriting of a 10-year-old boy with DCD (top) and a typically developing aged matched peer (bottom). The circles indicate pauses during writing. Note the location of the pauses are within words which is an indication of a lack of automaticity and poor skill acquisition. The typically developing sample demonstrates between word pauses only, which normally occur when a writer lifts the pen to transition between words. It is important to note that handwriting style (joined versus un-joined) is not a factor here as within word pauses occur in the handwriting of children with DCD irrespective of this. 
Papers of particular interest, published recently, have been highlighted as:

- Of importance

•- Of major importance

1. American Psychiatric Association. DSM-5. Diagnostic and Statistical Manual of Mental Disorders. 5th ed. Washington, DC: American Psychiatric Association; 2013.

2. Wilson P, Smits-Engelsman B, Caeyenberghs K, Steenbergen B, Sugden D, Clark J, et al. Cognitive and neuroimaging findings in developmental coordination disorder: new insights from a systematic review of recent research. Dev Med Child Neurol. 2017;59(11):1117-29.

3. Lingham R, Hunt L, Golding J, Jongmans M, Emond A. Prevalence of Developmental Coordination Disorder Using the DSM-IV at 7 Years of Age: A UK Population-Based Study. Pediatrics. 2009;123(4).

**4. Blank R, Barnett A, Cairney J, Green D, Kirby A, Polatajko H, et al. International clinical practice recommendations on the definition, assessment, intervention and psychosocial aspects of developmental coordination disorder. Developmental Medicine and Child Neurology 2019;61:242-85.

These international consensus and evidence-based recommendations include valuable material on handwriting assessment and intervention principles.

5. Wilson B, Neil K, Kamps P, Babcock S. Awareness and knowledge of developmental coordination disorder among physicians, teachers and parents. Child: care, health and development. 2013;39(2):296-300.

6. Missiuna C, Pollock N, Levac D, Campbell, W., Sahagian Whalen S, et al. Partnering for Change: An innovative school-based occupational therapy service delivery model for children with developmental coordination disorder. Canadian Journal of Occupational Therapy.79(1):41-50.

7. Berninger V, Amtmann D. Preventing written expression disabilities through early and continuing assessment and intervention for handwriting and/or spelling problems: Research into practice. In: Swanson H, Harris K, Graham S, editors. Handbook of learning disabilities. New York: Guilford Press; 2003. p. 323- 44.

8. McCutchen D. A capacity theory of writing: working memory in text composition. Educational Psychology Review. 1996;8:299-35.

9. Kellog R. A model of working memory in writing. In: Levy C, S., Ransdell, editors. The science of writing Mahwah, NJ: Lawrence Erlbaum Associates; 1996. p. 57-71.

10. Berninger V, Swanson L. Modifying Hayes \& Flower's model of skilled writing to explain beginning and developing writing. In: Butterfield E, editor. Children's writing: Toward a process theory of development of skilled writing. Greenwich, CT: JAI Press; 1994. p. 57-81.

11. Connelly V, Dockrell J, Walter K, Critten S. Predicting the quality of composition and written language bursts from oral language, spelling and handwriting skills in children with and without specific language impairment. Written Communication 2012;29:278-302.

12. Sumner $E$, Connelly $V$, Barnett $A$. The influence of spelling ability on handwriting production: Children with and without Dyslexia. Journal of Experimental Psychology: Learning, Memory, and Cognition. 2014;40(5):1441-7.

13. Olive T, Favart M, Beauvais L. Children's cognitive effort and fluency in writing: Effects of genre and of handwriting automatisation. Learning and Instruction. 2009;19(4):299-308.

14. Prunty M, Barnett A, Wilmut K, Plumb M. The Impact of Handwriting Difficulties on Compositional Quality in Children with Developmental Coordination Disorder. British Journal of Occupational Therapy. 2016;79(10):591-7.

15. Longstaff MG, Heath RA. The influence of motor system degradation on the control of handwriting movements: A dynamical systems analysis. Human Movement Science. 2003;22:91-110. 
16. Plamondon R, O'Reilly C, Remi C, Duval T. The lognormal handwriter: Learning, performing and declining. Frontiers in Psychology.4:1-14.

17. van Galen G. Handwriting: Issues for a psychomotor theory. Human Movement Science.10:165-91.

18. Sumner E, Barnett AL. Handwriting. In: Hopkins B, Geangu E, Linkenauger S, editors. The Cambridge Encyclopedia of Child Development Second Edition Cambridge: Cambridge University Press.; 2017. p. 530-6.

19. Rosenblum S, Margieh J, Engel-Yeger B. Handwriting features of children with developmental coordination disorder - results of triangular evaluation. Research in Developmental Disabilities. 2013(11):4134-41.

20. Rosenblum S. Development, reliability, and validity of the Handwriting Proficiency Screening Questionnaire (HPSQ). American Journal of Occupational Therapy. 2008;62(3):298-307.

21. Dunford C, Missiuna C, Street E, Sibert J. Children's Perceptions of the Impact of Developmental Coordination Disorder on Activities of Daily Living. British Journal of Occupational Therapy. 2005;68(5):207-14.

22. Clifford O'Brien J, Bergeron A, Duprey H, Olver C, St. Onge H. Children With Disabilities and Their Parents' Views of Occupational Participation Needs. International applications of the Model of Human Occupation in Mental Health. 2009;25(2):164-80.

23. Gaines R, Missiuna C. Early identification: are speech/language impaired toddlers at increased risk for developmental coordination disorder? Child: Care, Health and Development. 2007;33:325-32.

24. Kandel S, Soler O, Valdois S, Gros C. Graphemes as motor units in the acquisition of writing skills. . Reading and Writing. 2006;19:313-37.

25. Sumner E, Prunty M, Barnett A, Connelly, V. The influence of spelling ability on handwriting production: Children with and without Dyslexia. DCD UK London2018.

26. Prunty M. Developmental Coordination Disorder: A Focus on Handwriting. Oxford: Oxford Brookes University; 2014.

27. Department for Education. National curriculum in England English programmes of study: key stages 1 and 22013 [

28. Prunty M, Barnett A, Wilmut K, Plumb M. Handwriting speed in children with developmental coordination disorder: Are they really slower? Research in Developmental Disabilities.

2013;34(9):2927-36.

29. Prunty $M$, Barnett $A$, Wilmut $K$, Plumb $M$. An examination of writing pauses in the handwriting of children with Developmental Coordination Disorder. Research in Developmental Disabilities. 2014;35(11):2894-905.

30. Rosenblum S, Livneh-Zirinski M. Handwriting process and product characteristics of children diagnosed with developmental coordination disorder. Human Movement Science. 2008;27(2):20014.

31. Prunty M, Barnett A. Understanding handwriting difficulties: A comparison of children with and without motor impairment. Cognitive Neuropsychology 2017;34(3/4):205-18. .

*32. Prunty M, Barnett A. Accuracy and consistency of letter formation in children with Developmental Coordination Disorder: an exploratory study. Journal of Learning Disabilities. 2019;53(2):120-130.

This is the first published work to provide a detailed analysis of errors and inconsistencies in letter formation in children with $D C D$ 
*33. Barnett AL, Prunty MM, Rosenblum S. Development of the Handwriting Legibility Scale (HLS): a preliminary examination of Reliability and Validity. Research in Developmental Disabilities. 2018;72:240-7.

The HLS offers a practical tool for the assessment of different components of legibility that can be used across different writing tasks, languages and scripts.

34. Bo J, Lee C. Motor skill learning in children with Developmental Coordination Disorder. . Research in Developmental Disabilities 2013;34(6):2047-55.

35. Rosenblum S, Gafni-Lachter L. Handwriting Proficiency Screening Questionnaire for Children (HPSQ-C): Development, Reliability, and Validity. The American Journal of Occupational Therapy. 2015.

36. Webb AM. The relationship between poor handwriting and written composition in children with developmental coordination disorder. London: Institute of Education University of London; 2013.

37. Wechsler D. Wechsler Individual Achievement Test - Third UK Edition (WIAT-III UK). London: Pearson Assessment; 2017.

38. Kaufman AS, Kaufman NL. Kaufman Test of Educational Achievement, Third Edition (KTEA3). . London: Pearson Assessment; 2014.

39. Robertson G, Wilkinson G. WRAT5: Wide Range Achievement Test (5th Edition). London, UK: Pearson; 2017.

40. Elliot CD, Smith P. British Ability Scales 3. London: GL Assessment; 2012.

41. Graham S, Harris KR. Improving learning disabled students' skills at composing essays: Selfinstructional strategy training. Exceptional Children. 1989.

42. Weintraub N, Drory-Asayag A, Dekel R, Jokobovits H, Parush, S. Developmental trends in handwriting performance among middle school children. OTJR: Occupation, Participation and Health. 2007.

43. Smits-Engelsman BCM, van Bommel-Rutgers I, Van Waelvelde H. Systematic Detection of Writing problems. Technical Manual SOS-2-EN. 2015.

44. Barnett A, Henderson SE, Scheib B, Schulz J. Detailed Assessment of Speed of Handwriting (DASH). London: Pearson Assessment; 2007.

45. Waelvelde van H, Hellinckx T, Peersman W, Smits-Engelsman BCM. SOS: A screening instrument to identify children with handwriting impairments. Physical \& Occupational Therapy in Pediatrics. 2012;32(3):306-19.

46. Alamargot D, Morin M. Does Handwriting on a Tablet Screen Affect Students' Graphomotor Execution? A Comparison Between Grades Two and Nine. Human Movement Science 2015;44:32-41. 47. Beery K, Beery N, Buktenica. The Beery-Buktenica developmental test of visual-motor integration (manual). Bloomington, MN: Pearson Assessments; 2010.

48. Prunty $M$, Barnett $A$, Wilmut $K$, Plumb M. Visual perceptual and handwriting skills in children with Developmental Coordination Disorder. Human Movement Science. 2016;49.

49. Prunty $M$. The role of pen grasp in the handwriting performance of children with Developmental coordination Disorder. 2020.

50. Camden C, Wilson B, Kirby A, Sugden E, Missiuna C. Best practice principles for management of children with developmental coordination disorder (DCD): results of a scoping review. Child: care, health and development. 2015;41(1):147-59.

51. Pollock N, Missiuna C. Perceived Efficacy and goal Setting System (PEGS) - 2nd Edition. Hamilton, ON: CanChild; 2015.

52. Cermak SA, Bissell J. Content and construct validity of Here's How I Write (HHIW): A child's self-assessment and goal setting tool. The American Journal of Occupational Therapy. 2014;68(3):296-306. 
**53. Santangelo T, Graham S. A comprehensive meta-analysis of Handwriting Instruction. Educational Psychology Review. 2016;28(2).

This paper reports an important meta-analysis of studies on handwriting instruction, indicating the evidence base available for intervention planning.

54. Zwicker JG, Montgomery I. Application of motor learning principles to handwriting instruction and intervention. Handwriting Today. 2012;11:9-19.

55. Campbell WN, Missiuna CA, Rivard LM, Pollock NA. "Support for everyone": Experiences of occupational therapists delivering a new model of school-based service. Canadian Journal of Occupational Therapy-Revue Canadienne D Ergotherapie. 2012;79(1):51-9.

**56. Campbell W, Camden C, Missiuna C. Reflections on Using a Community-Based and Multisystem Approach to Transforming School-Based Intervention for Children with Developmental Motor Disorders. Current Developmental Disorders Reports. 2016;3:129-37.

This paper reviews the innovative and practical Partnering for Change approach to intervention.

57. Polatajko $\mathrm{H}$, Mandich A. Enabling occupation in children: The cognitive orientation to daily occupational performance (CO-OP) approach. Ottawa: CAOT Publications ACE.; 2004.

58. Guadagnoli M, Lee T. Challenge point: A framework for conceptualizing the effects of various practice conditions in motor learning. Journal of Motor Behaviour. 2004;36(2):212-24. 59. Hoy MMP, Egan MY, Feder KP. A Systematic Review of Interventions to Improve Handwriting. 78. 2011(1):13-25. 